\title{
Characterization of circRNA-associated ceRNA networks in patients with nonvalvular persistent atrial fibrillation
}

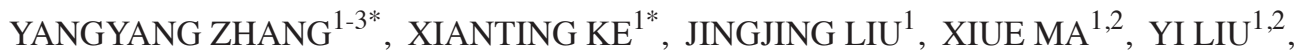 \\ DANDAN LIANG $^{1,2}$, LUXIN WANG ${ }^{1,4}$, CHANGFA GUO $^{5}$ and YIWEI LUO ${ }^{1,6}$ \\ ${ }^{1}$ Key Laboratory of Arrhythmias of The Ministry of Education of China; ${ }^{2}$ Research Center for Translational Medicine; \\ ${ }^{3}$ Department of Cardiovascular Surgery, East Hospital, Tongji University School of Medicine; \\ ${ }^{4}$ Shanghai East Hospital of Clinical Medical College, Nanjing Medical University, Shanghai 200120; \\ ${ }^{5}$ Department of Cardiac Surgery, Zhongshan Hospital, Fudan University, Shanghai 200032; \\ ${ }^{6}$ Department of Cardiology, East Hospital, Tongji University School of Medicine, Shanghai 200120, P.R. China
}

Received March 28, 2018; Accepted September 13, 2018

DOI: $10.3892 / \mathrm{mmr} .2018 .9695$

\begin{abstract}
Circular RNAs (circRNAs) are non-coding RNAs forming closed-loop structures, and their aberrant expression may lead to disease. However, the potential network of circRNA-associated competing endogenous RNA (ceRNA) involved in nonvalvular persistent atrial fibrillation (NPAF) has not been previously reported. In the present study, four left atrial appendages (LAA) of patients with NPAF and four normal LAAs were examined via RNA sequencing, and their potential functions were investigated via bioinformatics analysis. The circRNA-enriched genes were analyzed using Gene Ontology (GO) categories, while the enrichment of circRNAs was detected via the Kyoto Encyclopedia of Genes and Genomes (KEGG) database. A total of 296 significantly dysregulated circRNA transcripts were obtained, with 238 upregulated and 58 downregulated. A number of circRNAs were further confirmed using reverse transcription-quantitative polymerase chain reaction
\end{abstract}

Correspondence to: Dr Changfa Guo, Department of Cardiac Surgery, Zhongshan Hospital, Fudan University, 180 Fenglin Road, Shanghai 200032, P.R. China

E-mail: guo.changfa@zs-hospital.sh.cn

Dr Yiwei Luo, Key Laboratory of Arrhythmias of The Ministry of Education of China, East Hospital, Tongji University School of Medicine, 150 Jimo Road, Shanghai 200120, P.R. China

E-mail: yiwei_luo1977@163.com

*Contributed equally

Abbreviations: circRNA, circular RNA; ceRNA, competing endogenous RNA; NPAF, nonvalvular persistent atrial fibrillation; LAA, left atrial appendages; GO, Gene Ontology; KEGG, Kyoto Encyclopedia of Genes and Genomes; AF, atrial fibrillation; FC, fold-change; RT-qPCR, reverse transcription-quantitative polymerase chain reaction; TGF- $\beta 1$, transforming growth factor- $\beta 1$

Key words: NPAF, RNA-seq, circRNAs, ceRNA, pathway analysis. Furthermore, the more comprehensive circRNA-associated ceRNA networks were examined in patients with NPAF. GO categories and KEGG annotation analysis of circRNAs revealed that the circRNA-associated ceRNA networks were likely to influence AF though alterations in calcium and cardiac muscle contraction. The circRNA-associated ceRNA networks revealed that dysregulated circRNAs in NPAF may be involved in regulating hsa-microRNA (miR)-208b and hsa-miR-21. To the best of our knowledge, this study presents the circRNA-associated ceRNA networks in NPAF for the first time, which may have potential implications for the pathogenesis of AF. This study reveals a potential perspective from which to investigate circRNAs in circRNA-associated ceRNA networks (hsa_circRNA002085, hsa_circRNA001321) in NPAF, and provides a potential biomarker for AF.

\section{Introduction}

Atrial fibrillation (AF) is the most common type of cardiac arrhythmia in humans, characterized by irregular and rapid electrical activity of the atria $(1,2)$. In $2010, \mathrm{AF}$ affected $\sim 33.5$ million people, causing $\sim 5$ million new cases each year worldwide $(3,4)$. AF causes 130,000 mortalities/year in the USA (5) and affects $2-3 \%$ of the European population $(4,6,7)$. The number of patients with $\mathrm{AF}$ is predicted to increase rapidly in the coming decades and to gradually reduce quality of life $(8,9)$. In Asia, the number of patients with $\mathrm{AF}$ and $\mathrm{AF}$-associated stroke is estimated to reach 72 million and 2.9 million by 2050 , respectively $(3,10)$. Warfarin is commonly applied to treat nonvalvular persistent atrial fibrillation (NPAF) and research on NPAF has been extensive $(11,12)$, although the current treatment options remain inadequate. Thus, it is necessary to identify a novel biomarker for the prediction, diagnosis and treatment of NPAF.

Circular RNAs (circRNAs), non-coding RNAs identified in 1991 (13), regulate gene expression and act as microRNA (miRNA) 'sponges' by competing with endogenous RNA (ceRNA) networks to suppress specific miRNA activity $(14,15)$. circRNAs are associated with numerous diseases and may serve an important role in diagnosis or pathogenesis $(16,17)$. Previous 
studies have demonstrated that circRNA-miRNA-mRNA networks are likely to be involved in certain diseases $(18,19)$. However, there is little research regarding the functions of circRNAs in AF, particularly NPAF. The present study aimed to identify a novel biomarker for diagnosis of AF.

\section{Materials and methods}

Ethics approval statement. Written informed consent was obtained from patients prior to collection of left atrial appendages (LAAs), which were abandoned due to surgical techniques. The present study was conducted in accordance with the Declaration of Helsinki, and all experimental procedures were approved by the Ethics Committee of Shanghai East Hospital (approval no. 040-2017) as per relevant guidelines and regulations (clinical research registration no. ChiCTR-RRC-17014230).

Clinical specimens. A total of four male patients with NPAF without valvular disease as an AF group, and four healthy male organ donors as a control group, were recruited between January 2016 and December 2017. AF specimens were collected from the LAAs of patients with NPAF during LAA excision along with surgical atrial fibrillation ablation. Normal LAAs were collected from healthy donors. Excised LAAs were stored in RNA later in $-20^{\circ} \mathrm{C}$ prior to RNA extraction. Characteristics of the four patients are listed in Table I.

RNA extraction and qualification. Total RNA was isolated using TRIzol ${ }^{\circledR}$ reagent (Invitrogen; Thermo Fisher Scientific, Inc., Waltham, MA, USA), according to the manufacturer's protocol, and dissolved in RNase-free water. RNA purity was assessed using a Nanodrop ND-2000 device (Thermo Fisher Scientific, Inc., Wilmington, DE, USA) with an absorbance (A)260/A280 of 1.8-2.0, and integrity was assessed using an Agilent 2100 Bioanalyzer (Agilent Technologies, Inc., Santa Clara, CA, USA). The RNA integrity values of the RNAs obtained from the eight LAA specimens were 7.9, 7.7, 7.1, 7.3, $6.8,7.2,8.1$ and 7.5 , respectively.

RNA sequencing. Sequencing libraries were prepared as recommended by the VAHTS ${ }^{\text {TM }}$ Total RNA-seq (H/M/R) Library Prep kit (Illumina, Inc., San Diego, CA, USA). Ribosomal RNA was removed using target-specific probes, RNase $\mathrm{H}$ and DNA polymerase I (Finnzyme; Thermo Fisher Scientific, Inc., Waltham, MA, USA), and subsequently fragmented into pieces. Using reverse transcriptase and random primers, the RNA fragments were copied to the first strand of cDNA: 1 cycle of $25^{\circ} \mathrm{C}$ for $10 \mathrm{~min}$; and 1 cycle of $50^{\circ} \mathrm{C}$ for $15 \mathrm{~min}$ and $85^{\circ} \mathrm{C}$ for $5 \mathrm{~min}$, and the second strand was synthesized using DNA polymerase I, RNase H and dNTPs (dUTP, dATP, dGTP and dCTP; Invitrogen; Thermo Fisher Scientific, Inc.). Subsequently, single 'A' bases were added to the fragments of cDNA and then the adapter was ligated. To select the appropriate fragment size for sequencing, the library fragments were selected with VAHTSTM DNA Clean Beads (Vazyme Biotech Co., Nanjing, China). The second strand of cDNA was digested using an UDG (uracil-N-glycosylase) enzyme (Thermo Fisher Scientific, Inc.). Following cluster generation, 150-bp paired-end reads were produced by sequencing the libraries on the Illumina, Inc. Hiseq X10 platform.
Differential expression analysis. The expression levels of circRNAs were measured by RNA sequencing and expressed as 'transcripts per kilobase million'. Differentially-expressed circRNAs were analyzed using DESeq2 based on the negative binomial distribution test, and the thresholds were $\mathrm{P}<0.05$ and fold-change $(\mathrm{FC})>2$. P-values were calculated using a statistical algorithm (20).

Validation of circRNAs by reverse transcription-quantitative PCR (RT-qPCR). A number of differential circRNAs were randomly selected to test the accuracy of the RNA sequencing data by RT-qPCR. Total RNA was reverse transcribed into cDNA using PrimeScript ${ }^{\mathrm{TM}}$ RT Reagent kit (Takara Bio, Inc., Shiga, Japan) according to standard procedures. A total reaction volume of $20 \mu \mathrm{l}$, including 1,000 ng RNA, $4 \mu 1$ 5X PrimeScript RT Master Mix and RNase Free $\mathrm{dH}_{2} \mathrm{O}$. The thermocycling conditions were as follows: $37^{\circ} \mathrm{C}$ for $15 \mathrm{~min}$, then $10 \mathrm{sec}$ at $85^{\circ} \mathrm{C}$. RT-qPCR was performed with a SYBR Green kit (Promega Corporation, Madison, WI, USA) in the QuantStudio ${ }^{\mathrm{TM}} 6$ Flex system (Applied Biosystems; Thermo Fisher Scientific, Inc.), The reactions were incubated in 384 -well plates at $50^{\circ} \mathrm{C}$ for $2 \mathrm{~min}$, and $95^{\circ} \mathrm{C}$ for $10 \mathrm{~min}$, followed by 40 cycles of $95^{\circ} \mathrm{C}$ for $15 \mathrm{sec}$ and $60^{\circ} \mathrm{C}$ for $1 \mathrm{~min}$, followed by a dissociation curve. The internal reference was 18s rRNA (Applied Biosystems; Thermo Fisher Scientific, Inc.). Relative expression was quantified using the $2^{-\Delta \Delta \mathrm{Cq}}$ method (21). The primers are listed in Table II.

Gene ontology (GO) and kyoto encyclopedia of genes and genomes (KEGG) enrichment analysis. The differential or target genes of circRNAs were estimated and determined using GO enrichment analysis at the perl module (GO: TermFinder, http://search.cpan.org/dist/GO-TermFinder/) The KEGG enrichment of target genes of differential circRNAs was tested using $\mathrm{R}$ functions (22) (P hyper and Q value). The significantly-enriched GO terms and KEGG pathways met the criterion of corrected $\mathrm{P}<0.05$.

circRNA-ceRNA interaction prediction. The circRNA-miRNA interactions were predicted using TargetScan 6.0 (http://www. targetscan.org/vert_60/), which identifies miRNA targets and determines whether or not a given target is conserved across a given set of species. The sequences of circRNAs were predicted as miRNA binding seed sequence sites using potential miRNA response elements.

Statistical analysis. Statistical analyses was conducted using SPSS 19.0 software (IBM Corp., Armonk, NY, USA). When comparing two groups of profile differences, the fold-change (i.e. the ratio of the group averages) between the groups for each circRNA was computed. The differential expression of circRNAs was assessed using a Student's unpaired t-test. Error bars in the figures represent the means \pm standard deviation. $\mathrm{P}<0.05$ was considered to indicate a statistically significant difference.

\section{Results}

Overview of RNA-seq. No significant between-group difference was identified in the expression levels of 15,777 circRNAs. In total, 296 differential circRNAs were identified with $\mathrm{FC}>2$ and $\mathrm{P}<0.05$. Among them, 238 were 
Table I. Demographic characteristics of patients.

\begin{tabular}{|c|c|c|c|c|c|c|c|c|}
\hline No. & $\begin{array}{l}\text { Age, } \\
\text { years }\end{array}$ & Sex & NYHA & INR & $\begin{array}{l}\text { Coronary } \\
\text { artery }\end{array}$ & $\begin{array}{l}\text { Disease } \\
\text { complications }\end{array}$ & $\begin{array}{l}\text { Duration of } \\
\text { AF, years }\end{array}$ & $\begin{array}{l}\text { Drug } \\
\text { treatments }\end{array}$ \\
\hline 1 & 64 & Male & II-III & 3.38 & Negative & $\begin{array}{l}\text { Type II } \\
\text { diabetes }\end{array}$ & 2 & $\begin{array}{l}\text { Warfarin, furosemide, spironolactone } \\
\text { and Cordarone }\end{array}$ \\
\hline 2 & 76 & Male & II-III & 0.92 & Negative & Negative & 3 & $\begin{array}{l}\text { Warfarin, Cordarone, spironolactone, } \\
\text { furosemide and Betaloc }\end{array}$ \\
\hline 3 & 69 & Male & II-III & 2.03 & Negative & Negative & 4 & $\begin{array}{l}\text { Warfarin, Betaloc, furosemide, } \\
\text { spironolactone and Cordarone }\end{array}$ \\
\hline 4 & 60 & Male & II-III & 2.00 & Negative & Negative & 2 & Warfarin, furosemide and spironolactone \\
\hline
\end{tabular}

NYHA, New York Heart Association; INR, International Normalized Ratio; AF, atrial fibrillation.

Table II. The list of circRNAs qRT-PCR primers between NPAF and controls.

\begin{tabular}{lll}
\hline CircRNA & \multicolumn{1}{c}{ Forward primer } & Reverse primer \\
\hline hsa_circRNA011430 & CGAGCAGTACCCCACAATGG & TCTGTTGGCATGCTGCTGAA \\
hsa_circRNA015317 & ATTAGGCAGACTCTTCAAAACGC & AAACCCCCACCCACAAAGCA \\
hsa_circRNA016587 & TAGGCACAGCTCCTCCAGAT & TGTGAGATGCTTCACTGCATTC \\
hsa_circRNA003585 & GCTGCCCAATGATCTGCTTG & CCCTGCTTGCAGCTGTAGAAT \\
hsa_circRNA015019 & GGAGCCAAAGCCTAATCCGC & CTGCTGCCAAGGCATACTCA \\
hsa_circRNA003126 & ATGGACTGGCGGATCAAGGA & TGGCCACCAGTCACAAGGTA \\
\hline
\end{tabular}

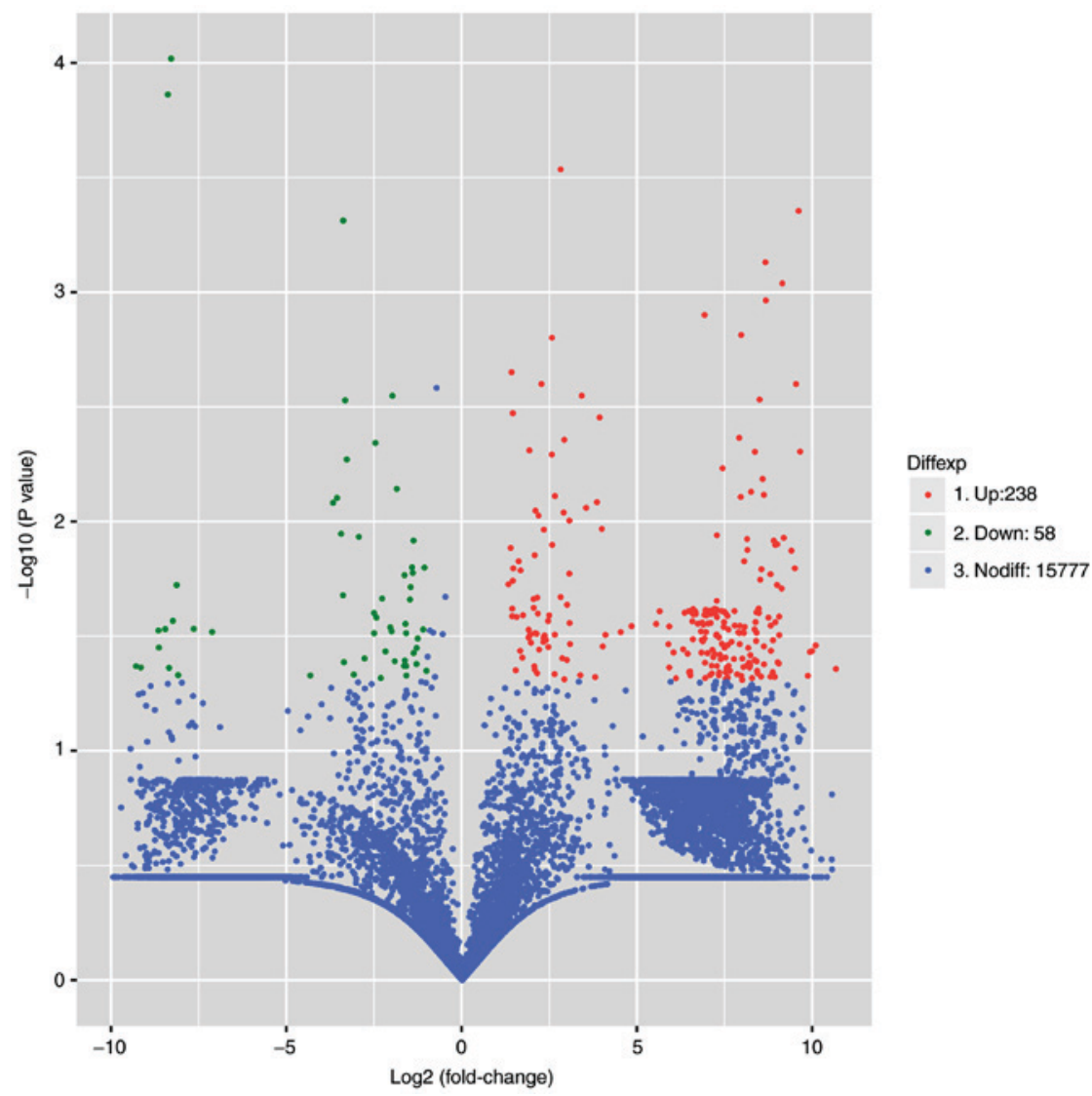

Figure 1. Volcano plot of circRNAs between the NPAF and control groups illustrates the distribution and number of circRNAs. Red and green represents upregulated and downregulated circRNAs, respectively, with fold-change $>2$ and $\mathrm{P}<0.05$. Blue represents unaltered circRNAs. NPAF, nonvalvular persistent atrial fibrillation; circRNA, circular RNA. 


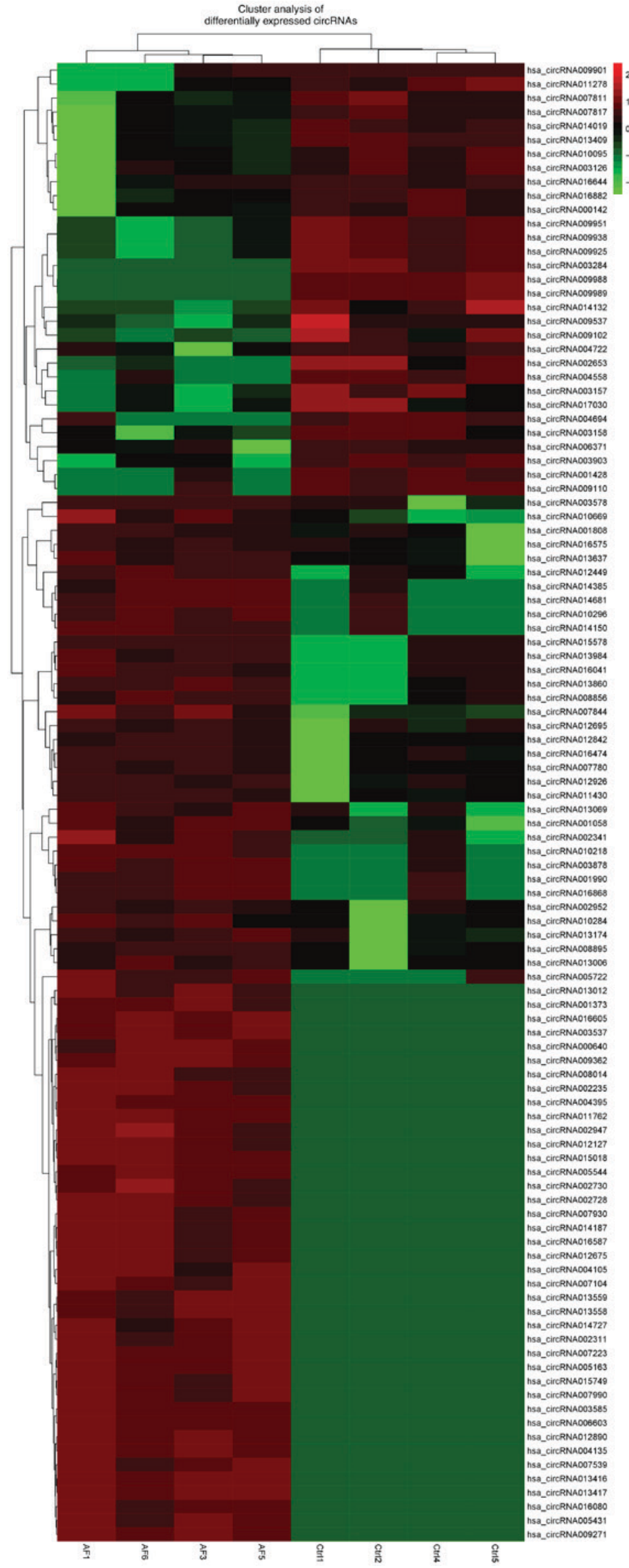

Figure 2. Heatmap of differentially-expressed circRNAs between the NPAF and control groups. The expression levels of circRNAs are represented by colored bars. The red and green color represents upregulated and downregulated circRNAs, respectively. Higher intensity of color indicates a greater degree of up- or downregulation. AF, NPAF; Ctrl, control. NPAF, nonvalvular persistent atrial fibrillation; circRNA, circular RNA. 
upregulated and 58 were downregulated in NPAF tissues compared with the controls (Figs. 1 and 2). The upregulated circRNAs (data not shown; available at https://1drv. ms/w/s!Al7sl_MjqbmWgQDki8ZjwTQd2j0H) with 'FC >2' and downregulated circRNAs (data not shown; available at https://1drv.ms/w/s!Al7sl_MjqbmWgQEKE-v8hCjzTevu) are listed. A number of the circRNAs were used in the subsequent analysis.

Validation of circRNAs by RT-qPCR. A total of six dysregulated circRNAs were randomly selected as representatives to validate the RNA sequencing data by RT-qPCR, and the primers are listed in Table II. All circRNAs were well-validated by qPCR, and the directions of the changes were consistent with the RNA-seq data (Fig. 3). The RT-qPCR data suggested the RNA-seq-identified circRNAs are reliable and require further research.

Functional enrichment analysis: $G O$ and KEGG pathway analysis. The differential circRNAs were annotated with GO, and were identified to be involved in the following functions: Cell component, biological process and molecular function (Fig. 4). GO analysis revealed that a number of functional pathways were enriched. Some of the top 10 terms were as follows: 'Voltage-gated calcium channel activity is involved in AV node cell action potential' in cell component GO (no. 0086056); 'cardiac muscle contraction is regulated by calcium ion signaling' in bioprocess GO (no. 0010882); 'release of sequestered calcium ion into cytosol is regulated by sarcoplasmic reticulum' in bioprocess GO (no. 0010880); and 'regulation of cardiac muscle cell action potential is involved in regulation of contraction' in bioprocess GO (no. 0098909), which were the most closely associated with AF. These criteria from the GO analysis were also applied to the KEGG enrichment analysis. The top 20 KEGG pathway analysis demonstrated that the most significant pathways involved in AF were 'arrhythmogenic right ventricular cardiomyopathy' (no. ko05412) and 'cardiac muscle contraction' (no. ko04260; Fig. 5).

Construction of circRNA-ceRNA interaction network. To analyze the interaction between differential circRNAs and ceRNAs, a ceRNA network in NPAF was investigated using RNA sequencing data. To examine which circRNAs were vital for NPAF progression, nine differential circRNAs were selected from those with calcium-associated parental genes. Complete sequences of hsa_circRNA-011785, -001321, $-003878,-002085,-003884,-003876,-007410,-007411$ and -004558 are listed in Table III. Details are provided in Table IV. A representative network of circRNAs and miRNAs is presented in Fig. 6. The interactions between hsa_circRNA002085 and hsa-miRNA (miR)-21, and between hsa_circRNA001321 and hsa-miR-1, suggest the possibility of an NPAF regulatory mechanism.

\section{Discussion}

In total, 296 differential circRNAs were identified between NPAF tissues and the controls $(\mathrm{FC}>2 ; \mathrm{P}<0.05)$, including 238 upregulated circRNAs and 58 downregulated circRNAs. To validate the RNA sequencing data, six representative

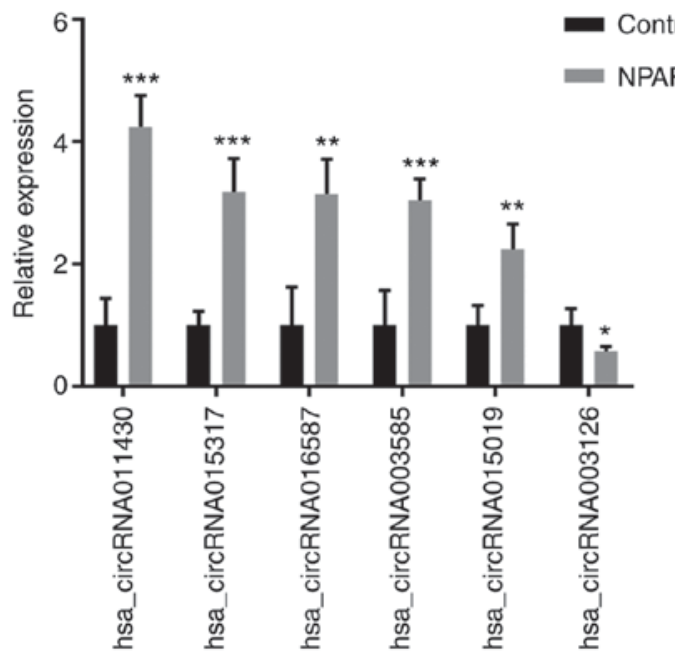

Figure 3. Validation of differentially expressed circRNAs between the NPAF and control groups by reverse transcription-quantitative polymerase chain reaction. A total of six circRNAs were validated and 18s was used as the internal control. The validation rate of the six circRNAs was approaching $100 \%$, demonstrating that the circRNA sequencing data were reliable. ${ }^{*} \mathrm{P}<0.05,{ }^{* *} \mathrm{P}<0.01,{ }^{* * *} \mathrm{P}<0.001$ vs. control. NPAF, nonvalvular persistent atrial fibrillation; circRNA, circular RNA.

dysregulated circRNAs (hsa_circRNA-011430, -015317, -016587, -003585, -015019 and -003126) were selected. The RT-qPCR results suggested that the RNA sequencing-identified circRNAs are reliable and merit further research. The significantly dysregulated circRNAs in patients with NPAF may serve a regulatory role in the mechanism of AF progression.

Unlike linear RNAs, circRNAs are covalently linked to form closed-loop structures without $5^{\prime}$ caps or $3^{\prime}$ tails $(18,23-25)$. In circRNAs, a downstream splice donor is joined by 'back-splicing' to the upstream splice acceptor $(17,26,27)$. circRNAs are associated with, and serve important roles in the diagnosis and pathogenesis of, numerous diseases $(16,17)$, including colorectal cancer $(28)$, breast cancer (29) and gastric cancer (30). Furthermore, circRNAs are able to upregulate the expression levels of fibrosis-associated genes in cardiac fibroblasts (31). Other studies have reported that a circRNA-ceRNA network may be present in certain diseases $(18,19)$. However, the circRNAs and circRNA-ceRNA network leading to AF remain to be elucidated.

$\mathrm{AF}$ remains a common cause of stroke, heart failure and cardiovascular mortality worldwide. AF may occur idiopathically, which is associated with familial inherent specific genetic mutations (32). Certain mechanisms leading to $\mathrm{AF}$ are primarily associated with the remodeling of ion channel functions, including those of $\mathrm{K}^{+}$channels, and cellular $\mathrm{Ca}^{2+}$ handling and release (33-36). Other mechanisms of AF include mutations and the abnormal expression of genes encoding cardiac ion channels, including potassium voltage-gated channel subfamily Q member 1, potassium voltage-gated channel subfamily $E$ regulatory subunit 2, potassium voltage-gated channel subfamily J member 2 (KCNJ2) and sarcoplasmic/endoplasmic reticulum calcium ATPase 2A (SERCA2) (36). 


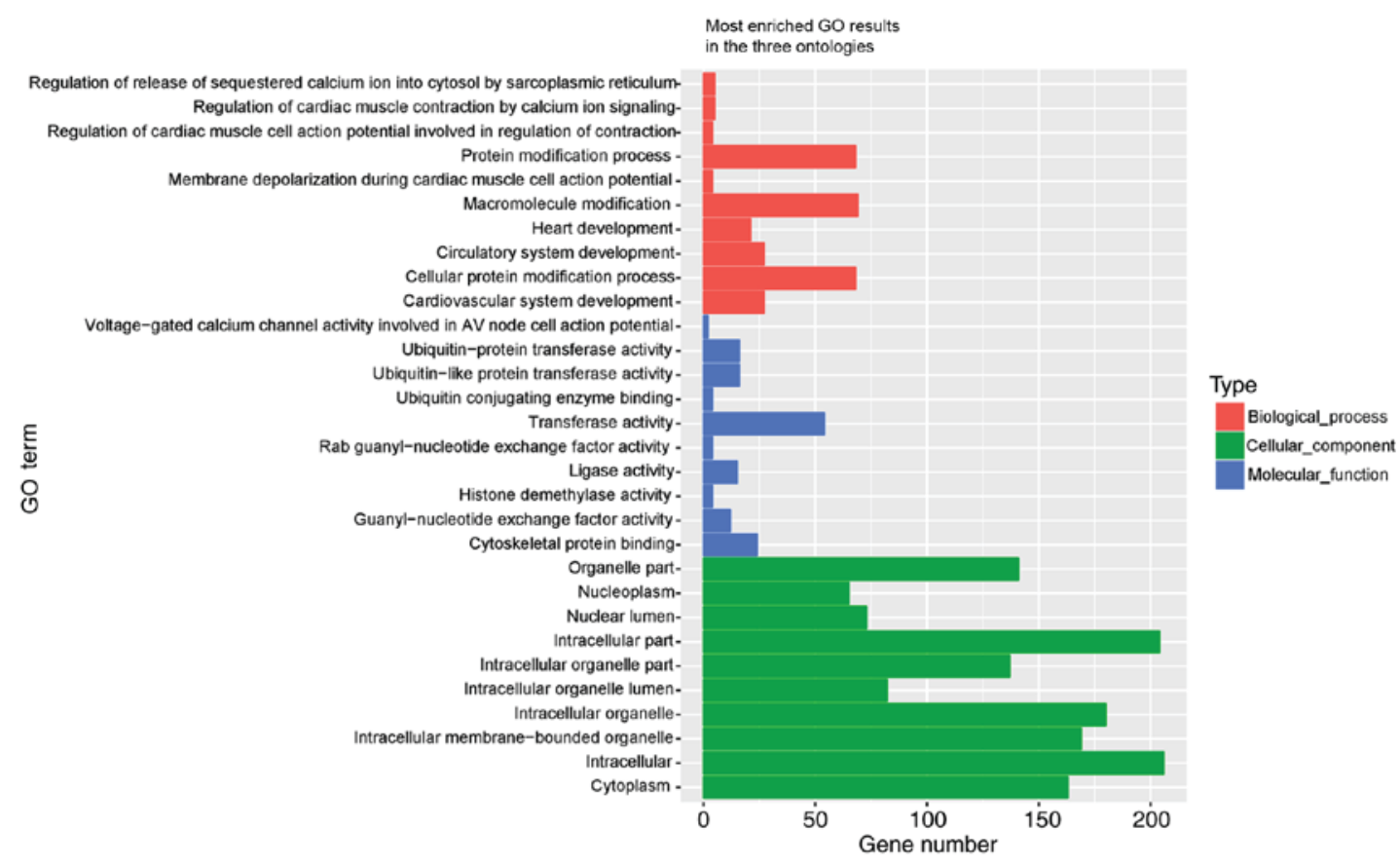

Figure 4. GO annotations and enrichment analysis of the differentially expressed circRNAs between the NPAF and control groups. The differentially expressed circRNAs between the NPAF and control groups were annotated with respect to three components (cellular component, biological process and molecular function). Q $<0.05$. GO, gene ontology; NPAF, nonvalvular persistent atrial fibrillation; circRNA, circular RNA.

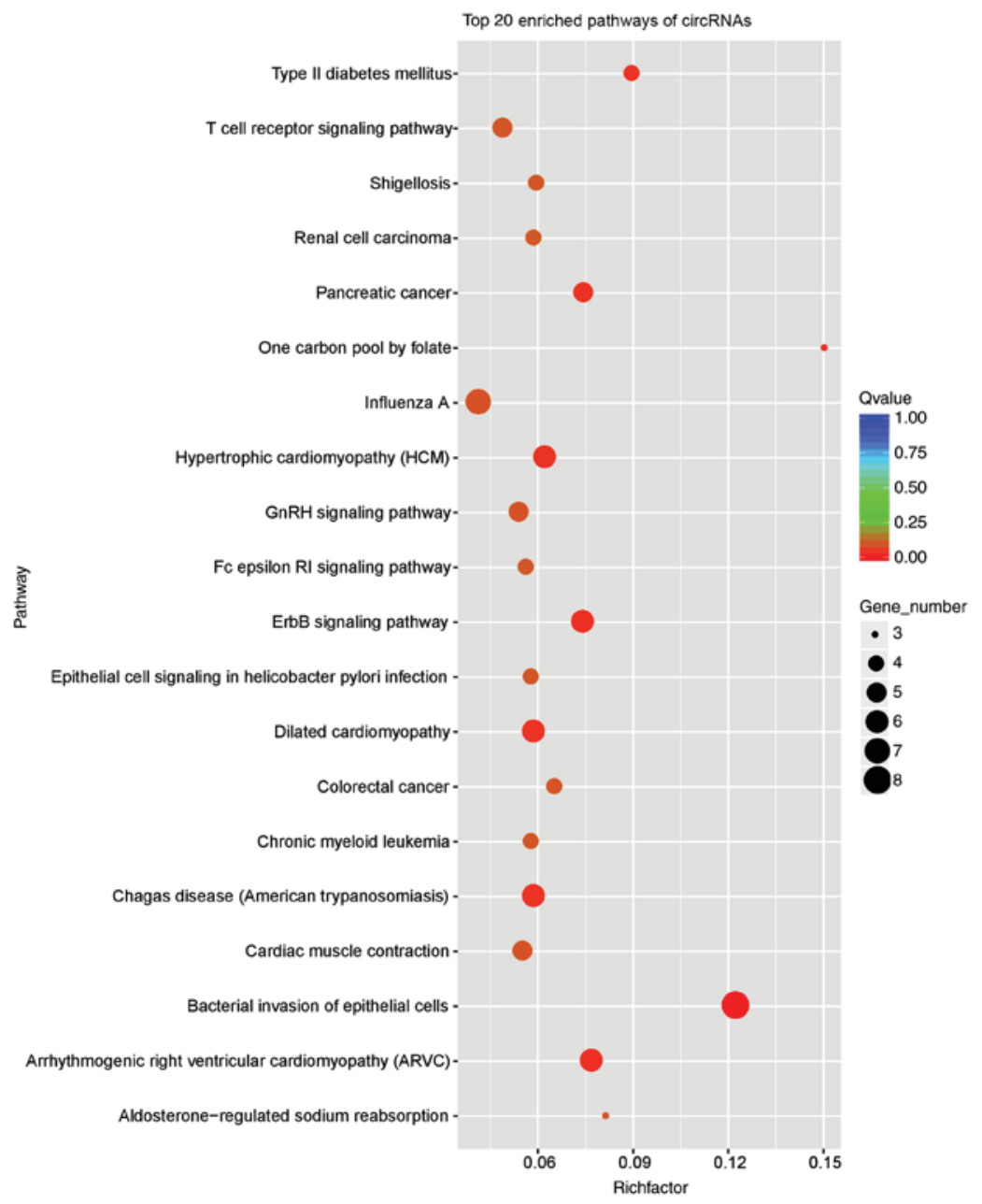

Figure 5. Kyoto Encyclopedia of Genes and Genomes pathway enrichment analysis of the differentially expressed circRNAs between the nonvalvular persistent atrial fibrillation and control groups. The number of genes is represented by the size of the circle, and the Q-value is represented by the color. circRNA, circular RNA. 

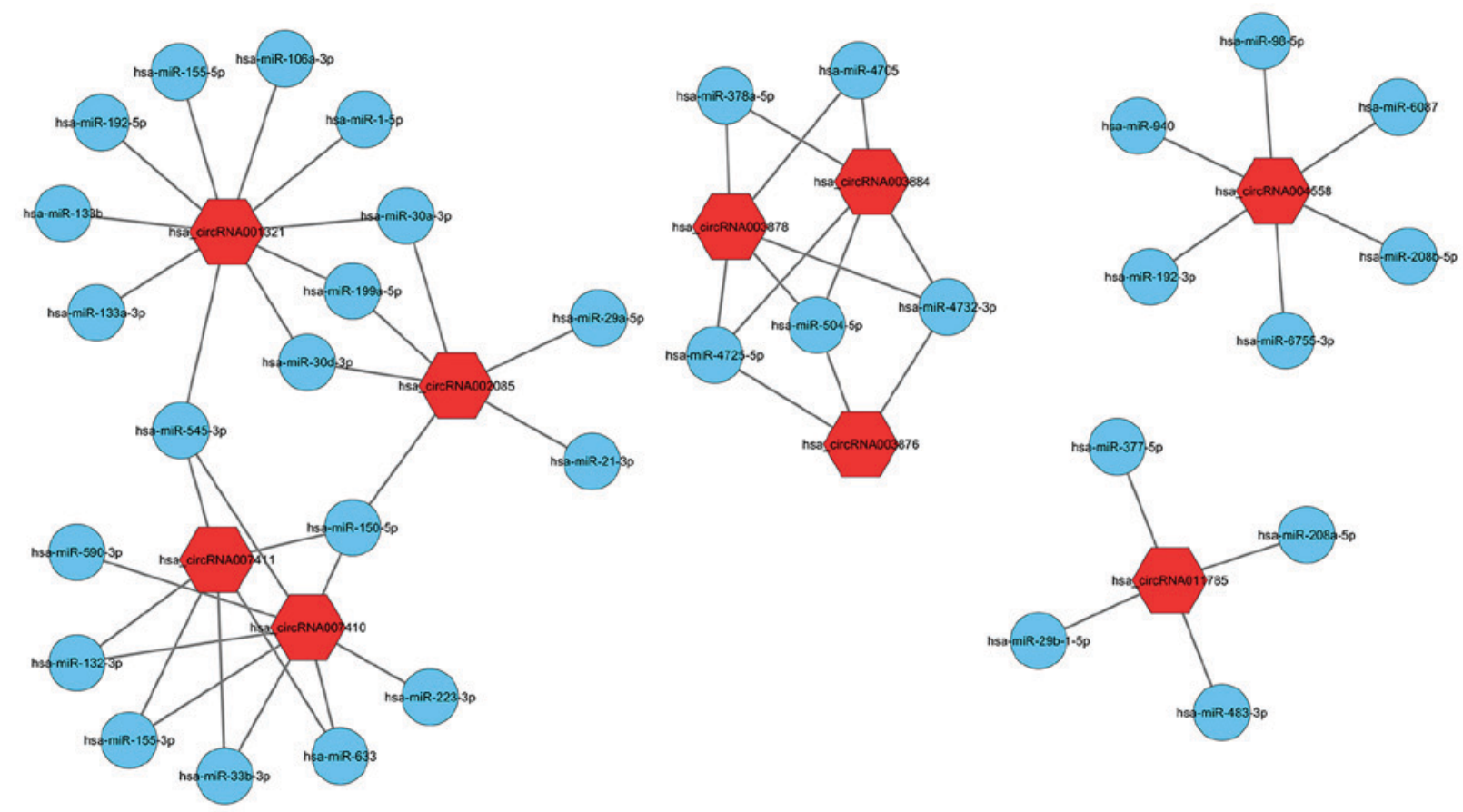

Figure 6. cirRNA-miRNA networks between the NPAF and control groups. Red and blue represents circRNAs and miRNAs, respectively. miRNA/miR, microRNA; circRNA, circular RNA.

For instance, calcium gene expression was identified to be abnormal in AF (37), and was hypothesized to contribute to the propensity for structural remodeling in AF. The calcium signaling pathway is essential in the electrical remodeling of $\mathrm{AF}$ and may induce the recurrence of $\mathrm{AF}(37)$. Abnormalities in intracellular $\mathrm{Ca}^{2+}$ handling are crucially involved in AF-initiated focal activity and perpetuation through rapidly firing foci and reentry (37).

GO analysis performed using differential circRNAs between the NPAF and control groups demonstrated that a number of functional pathways were enriched. The primary cell component was GO (no. 0086056) 'voltage-gated calcium channel activity is involved in AV node cell action potential'. The main bioprocesses were GO (no. 0010882) 'cardiac muscle contraction is regulated by calcium ion signaling', GO (no. 0010880) 'release of sequestered calcium ion into cytosol is regulated by sarcoplasmic reticulum' and GO (no. 0098909) 'regulation of cardiac muscle cell action potential is involved in regulation of contraction'. The specific genomes were 'CACNB2, CACNA1C' (GO:0086056); 'SLC8A1, ATP2A2, CACNA1C, ANK2, RYR2' (GO:0010882); 'DHRS7C, CACNA1C, SLC8A1, RYR2, ANK2' (GO:0010880); 'CACNA1C, ATP2A2, RYR2, ANK2' (GO:0098909). These genes are primarily involved in $\mathrm{Ca}^{2+}$ channels and may be the most closely associated with AF. A total of two of the most significant top 20 KEGG pathways were 'arrhythmogenic right ventricular cardiomyopathy' (no. ko05412) and 'cardiac muscle contraction' (no. ko04260), which were also associated with AF. Differential expression of circRNAs may be affected by $\mathrm{AF}$ and atrial remodeling. The present study identified a number of differential circRNAs in AF.

The circRNA-ceRNA interactions were constructed to examine which circRNAs serve important roles in NPAF progression. A total of nine differential circRNAs
(hsa_circRNA-011785, -001321, -003878, -002085, -003884, $-003876,-007410,-007411$ and -004558) from calcium-associated parental genes were selected. miRNAs serve multiple roles in atrial fibrillation, including regulating electrical remodeling by targeting the genes involved in different ion channels, and regulating structural remodeling in cardiac tissues by increasing cardiac fibrosis or apoptosis. Different miRNAs have been demonstrated to be upregulated or downregulated in patients with $\mathrm{AF}$ (21), and may target different genes to regulate cardiac function. Different miRNAs may target single genes and serve similar roles, including repressing $\mathrm{I}_{\mathrm{K} 1}$ (potassium current) by targeting $\mathrm{KCNJ} 2$ via miR-1 and miR-26 $(22,38)$. In the present study, certain circRNAs were also dysregulated in AF, which might indicate an association. The interactions between hsa_circRNA004558 and miR-208b, between hsa_circRNA002085 and hsa-miR-21, and between hsa_circRNA001321 and hsa-miR-1 may suggest a possible regulatory mechanism in AF. For instance, miR-208b was demonstrated to be upregulated in patients with $\mathrm{AF}$ and an ovine model. Furthermore, a high miR-208b level was demonstrated to increase MYH7 expression and alter the subcellular localization of connexin43 (39). miR-208b has been reported to reduce the expression levels of CaV1.2 and SERCA2, which further reduce L-type $\mathrm{Ca}^{2+}$ current density and sarcoplasmic reticulum $\mathrm{Ca}^{2+}$ load/release, respectively (39). These alterations are hallmarks of atrial remodeling during AF (40). miR-21 is reportedly associated with atrial fibrosis regulation in $\mathrm{AF}$, which inhibits the proliferation of cardiac fibroblasts by inactivating the transforming growth factor (TGF)- $\beta 1 /$ mothers against decapentaplegic homolog (Smad)2 signaling pathway (41).

miR-21-3p may regulate sepsis-associated cardiac dysfunction and the development of cardiac hypertrophy. When miR-21-3p is inhibited, such diseases may be treated via a protective strategy $(42,43)$. The upregulated inward 


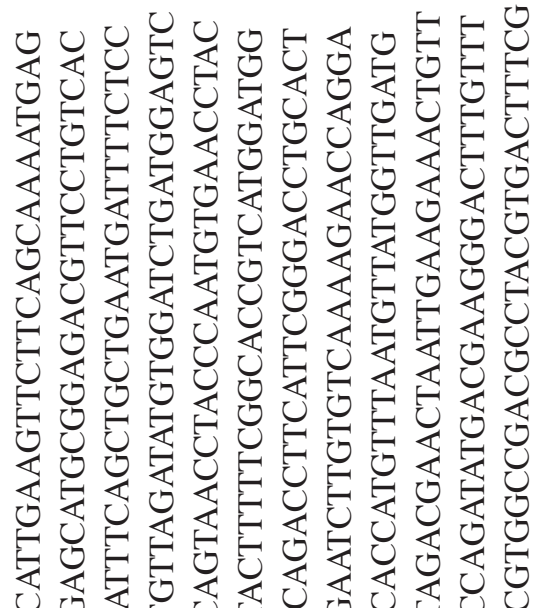

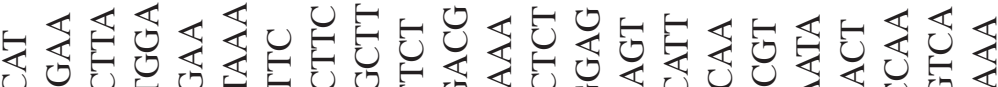

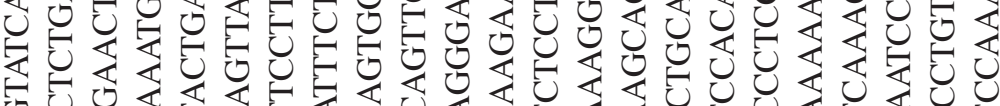

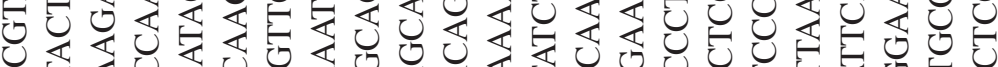

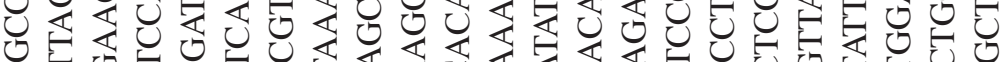

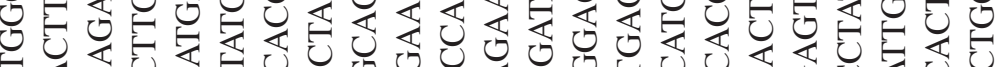

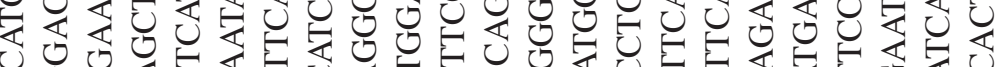

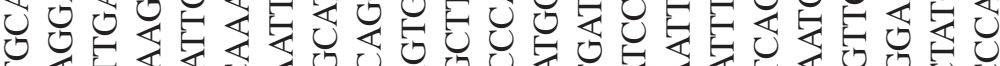

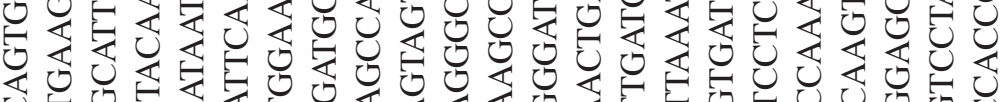

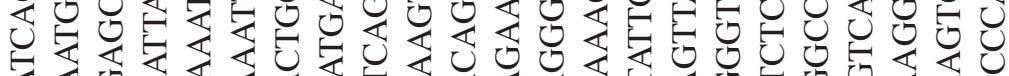

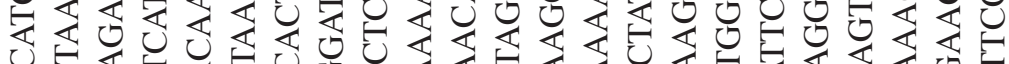

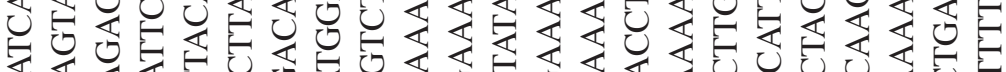

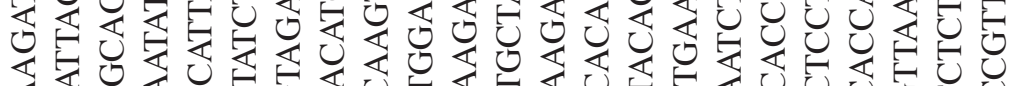

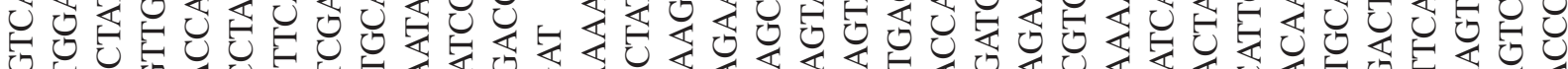

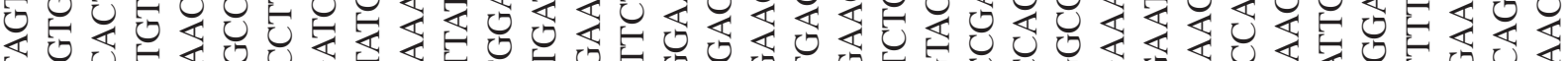

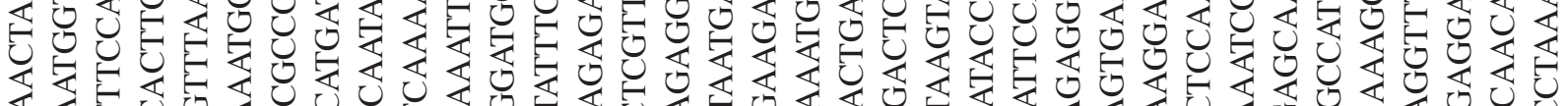

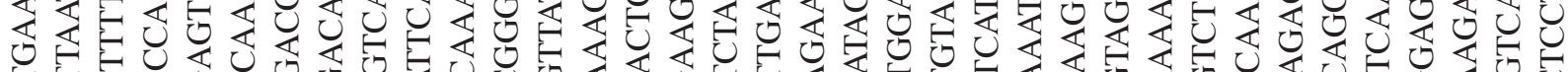

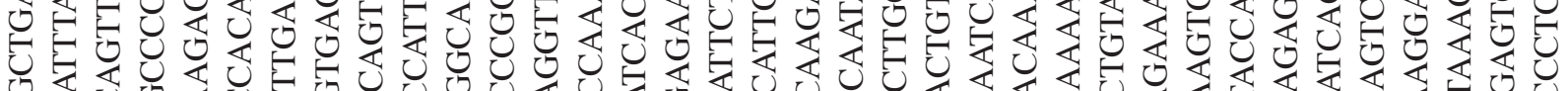

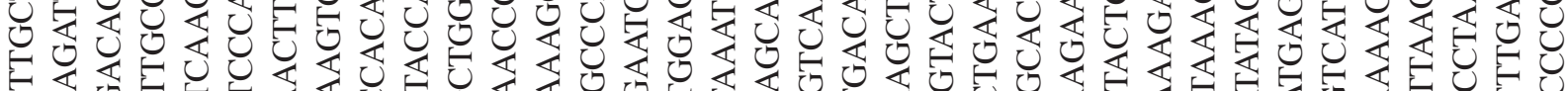

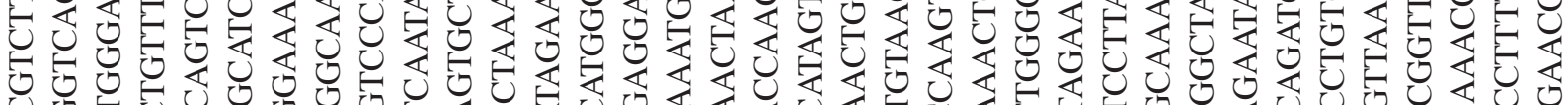
U

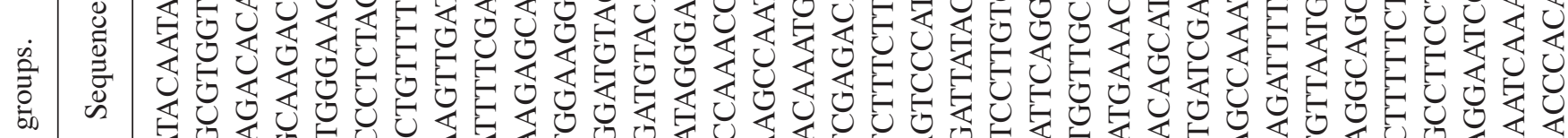

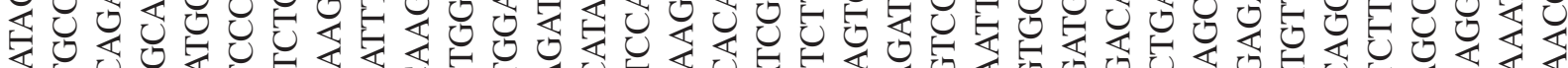

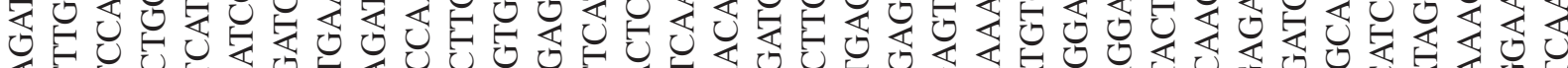

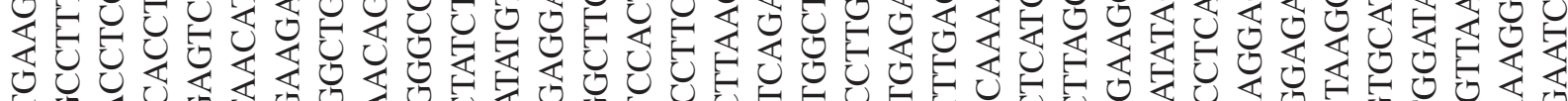

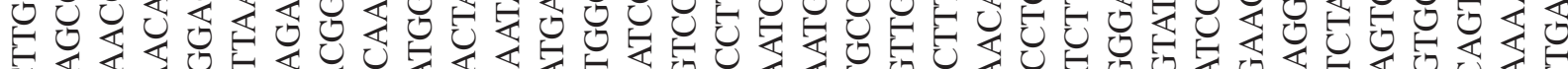

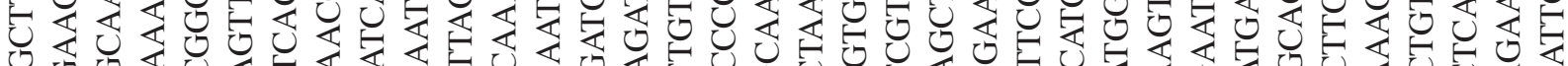

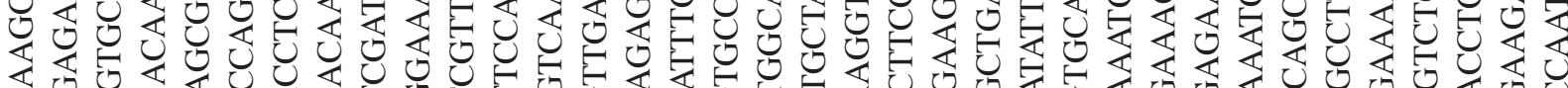

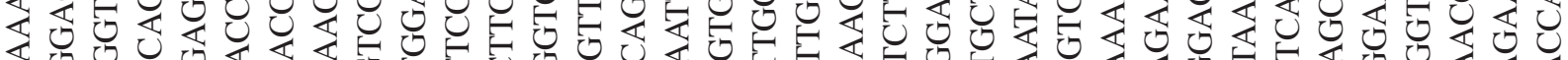

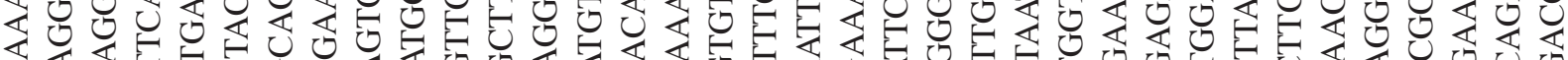

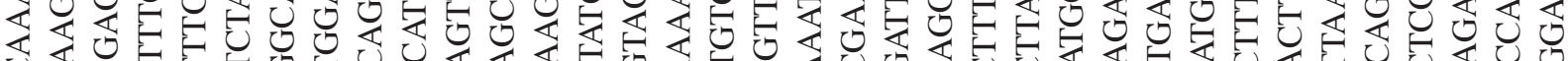

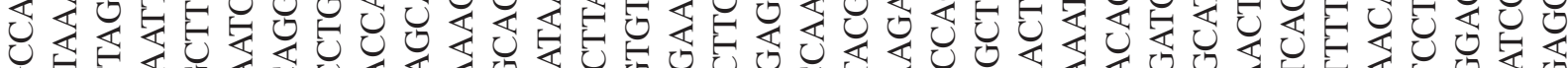

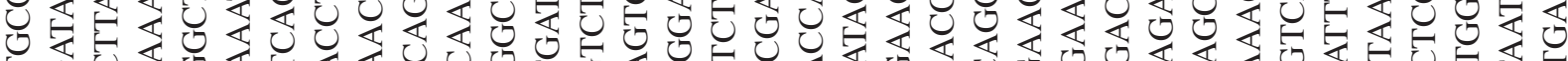

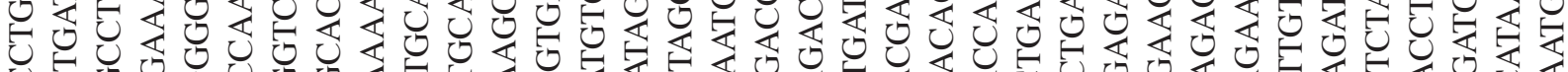
U

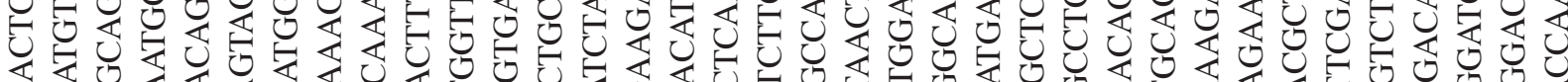

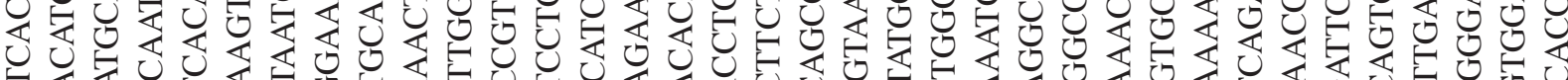

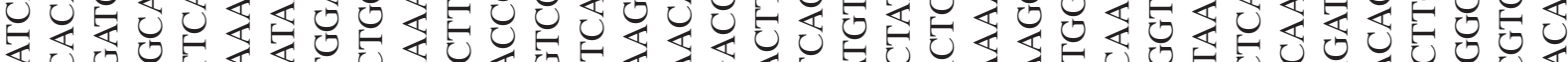

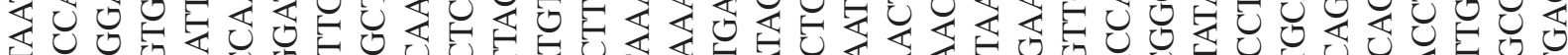

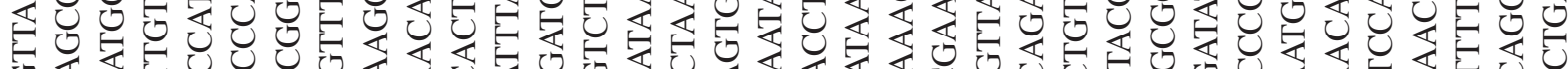

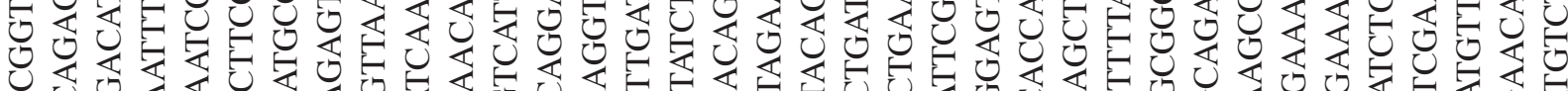

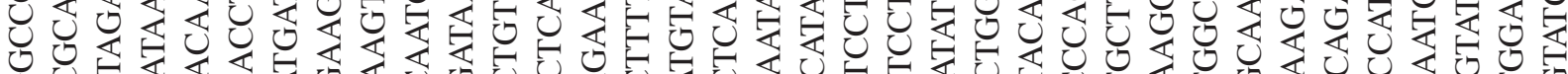

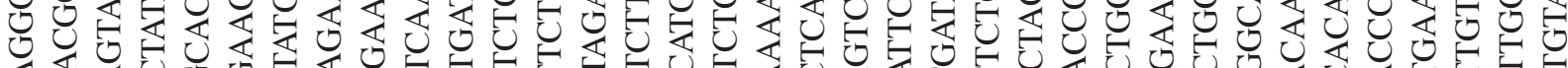

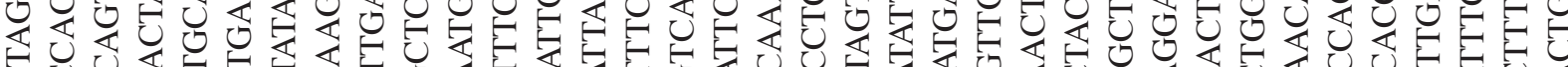

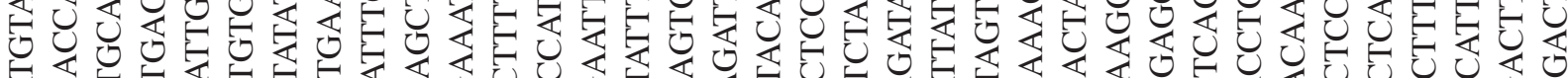

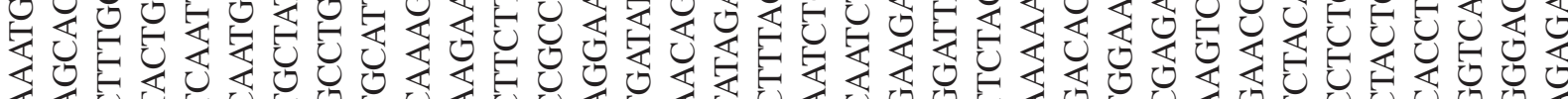

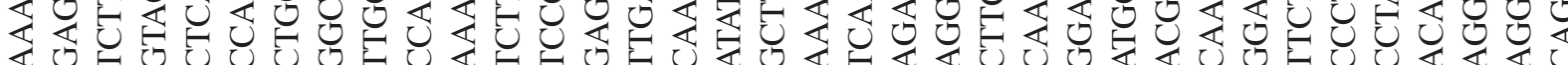

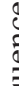




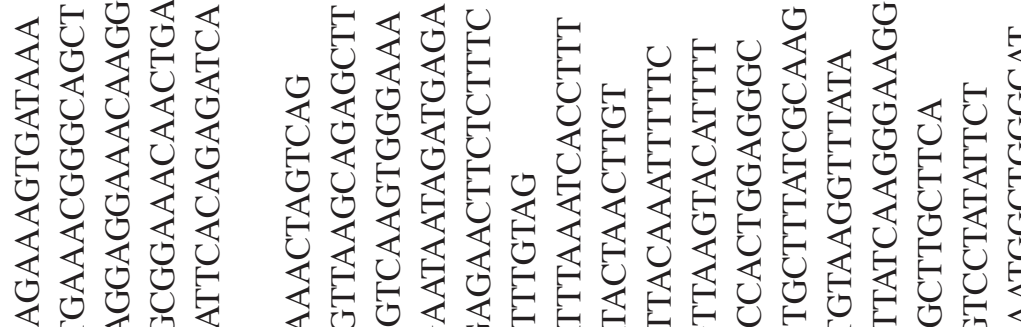

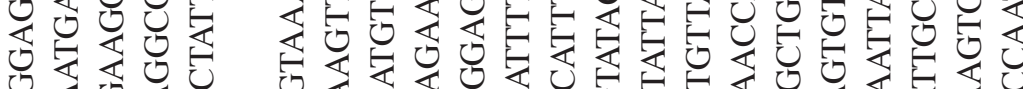

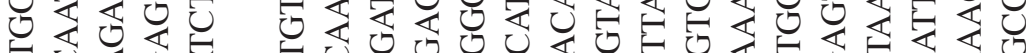

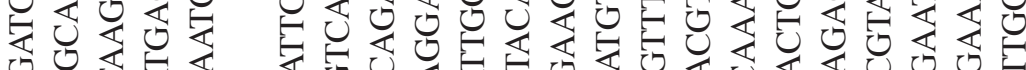

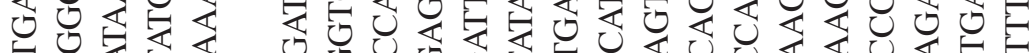
它它安

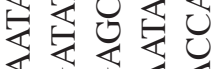
O

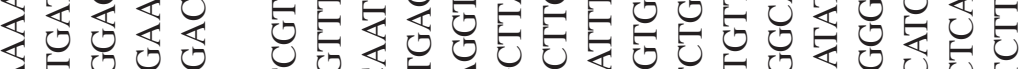

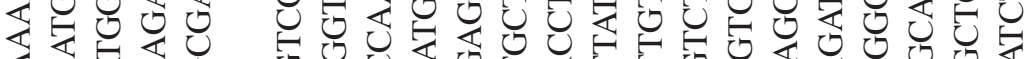

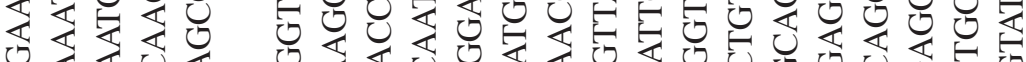

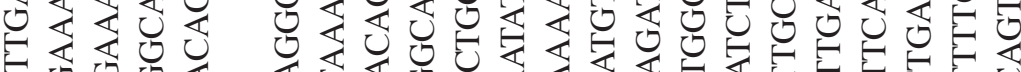

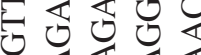

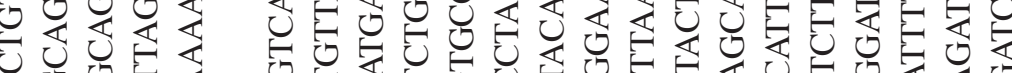
U 退这安

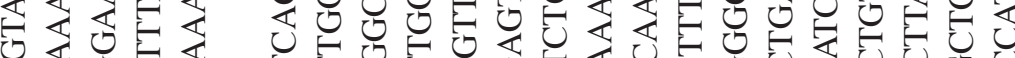

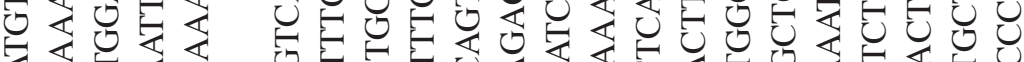

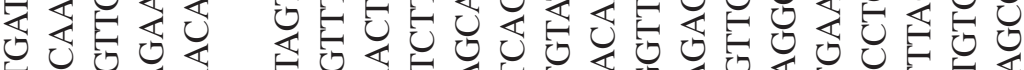
OU

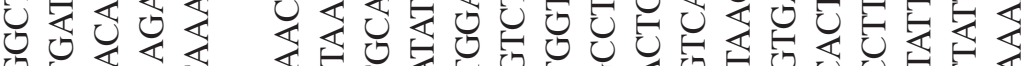

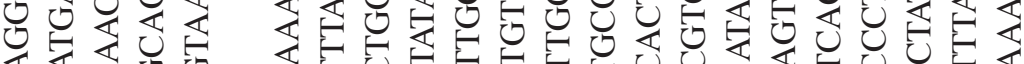

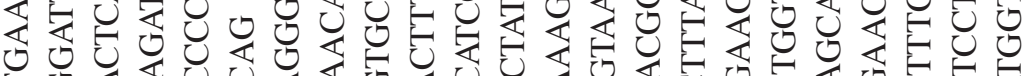

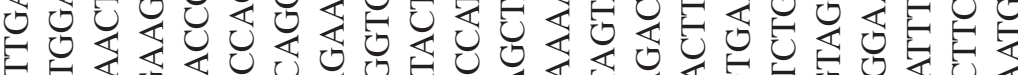

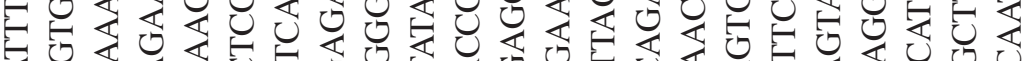

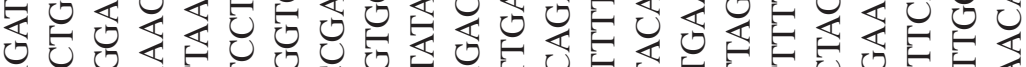

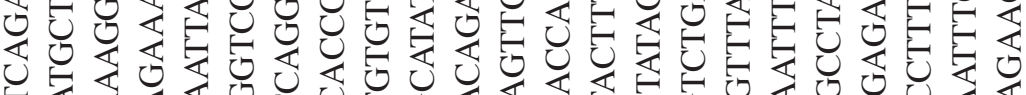

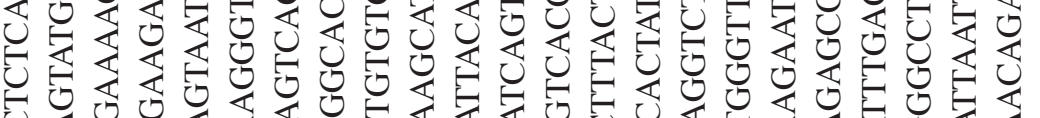

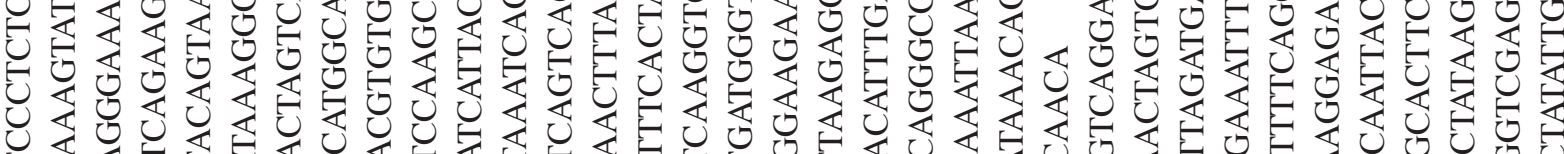

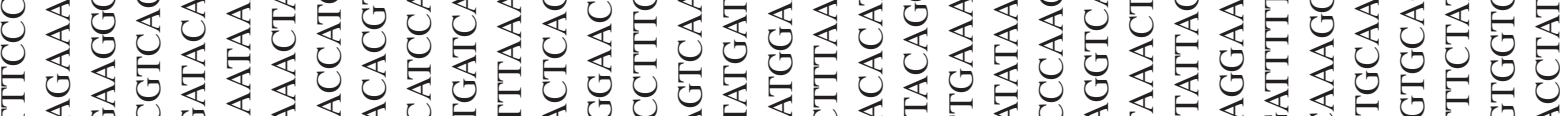

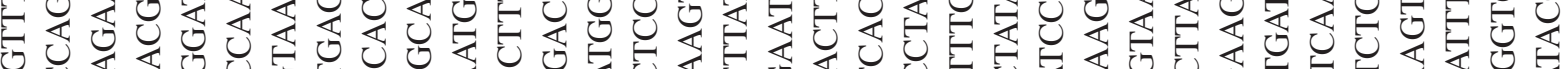

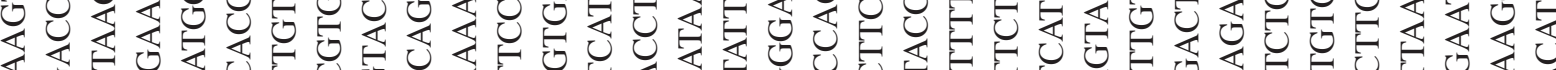

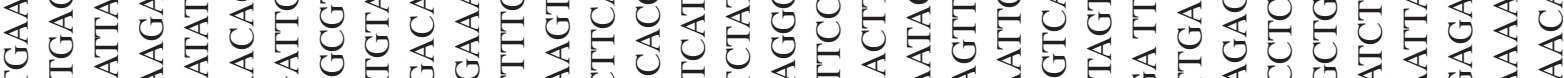

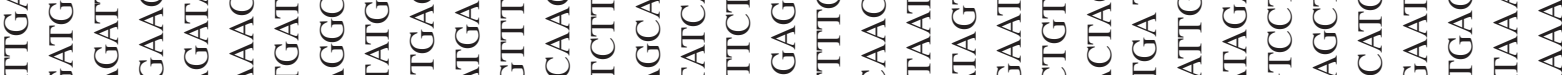

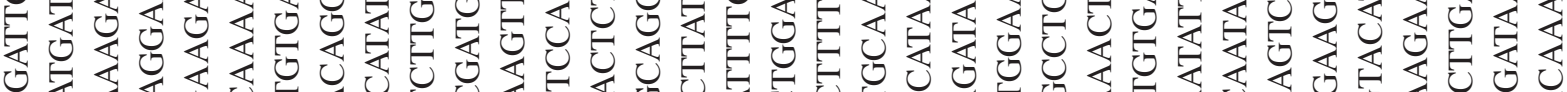

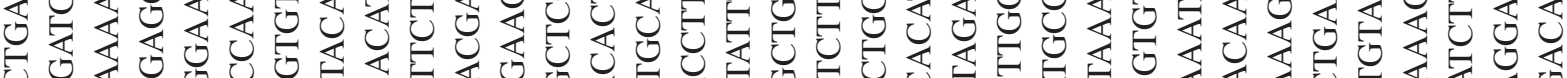

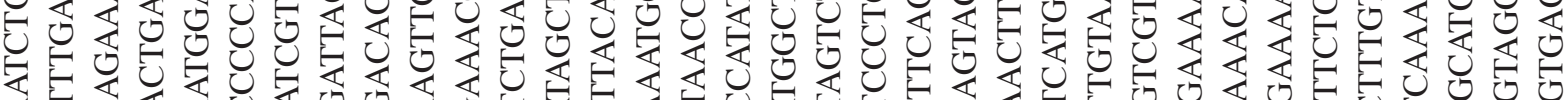

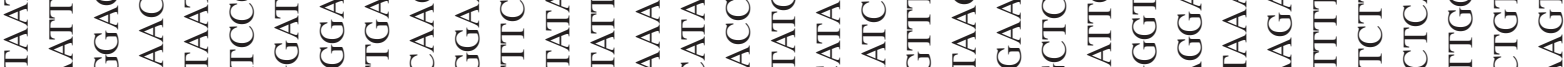

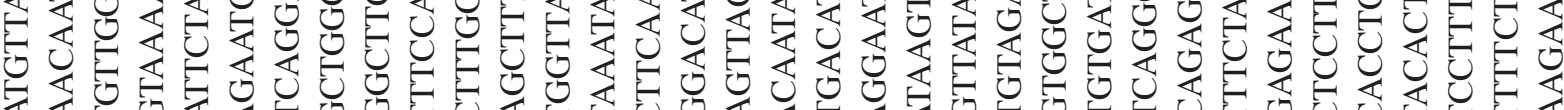

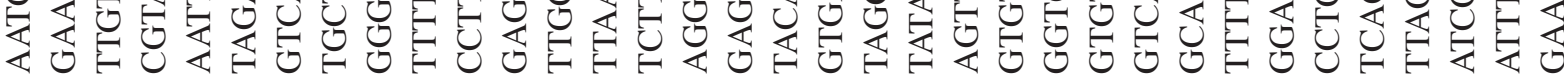




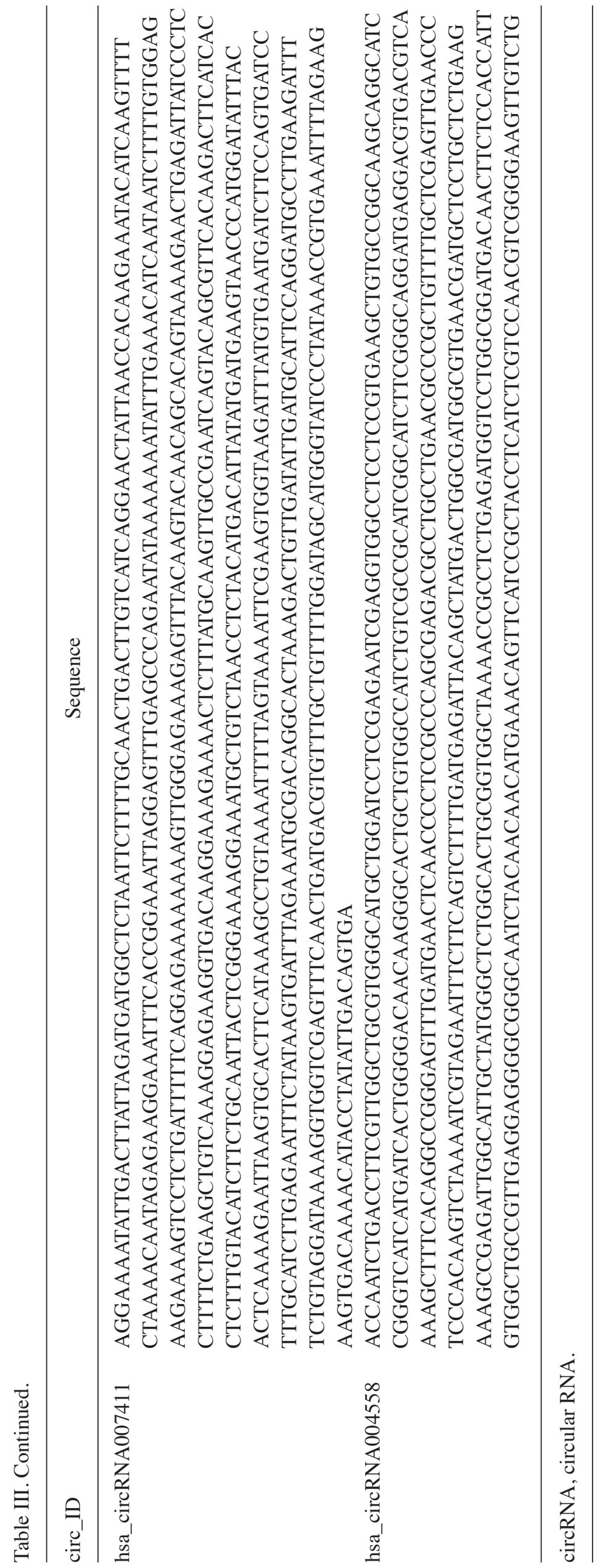




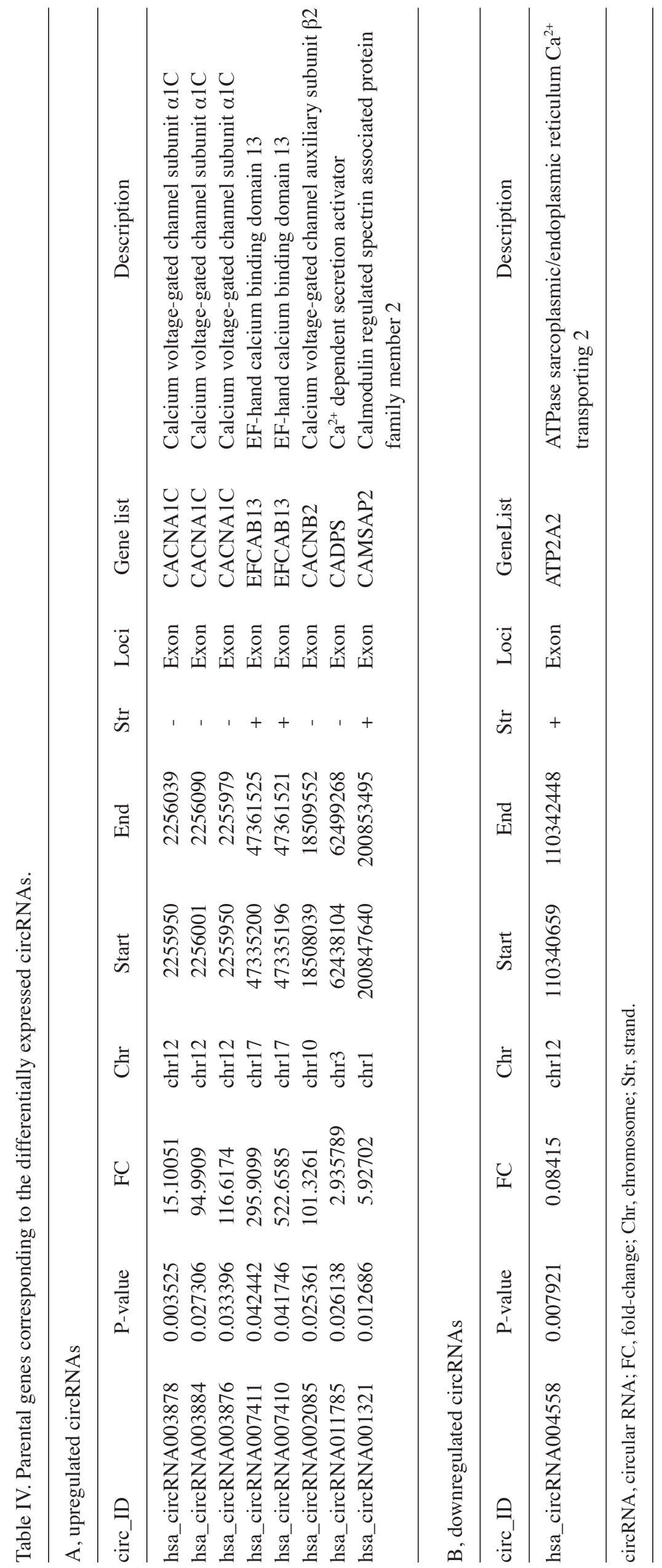


rectifier currents $\left(\mathrm{I}_{\mathrm{K} 1}\right)$ associated with the electrical remodeling of AF are required to maintain AF. Additionally, miR-1 expression is downregulated in patients with AF, which may increase the levels of inwardly rectifying potassium channel (Kir)2.1 subunits and $\mathrm{I}_{\mathrm{K} 1}(44,45)$. miR-4732-3p is targeted by three circRNAs (hsa_circRNA-003876,-003878 and -003884), and represses TGF- $\beta$ signaling by targeting Smad2 and Smad4, and promoting cell proliferation (46). The TGF- $\beta$ and Smad signaling pathways serve essential roles in atrial fibrosis.

The results of the present study provide a potential novel insight into the molecular mechanisms and therapeutic implications of AF. The circRNA-ceRNA interactions identified may act as biosignatures for AF, and provide evidence for identifying a novel agent for the diagnosis and gene-targeted therapy of AF.

The current study has a few limitations. Firstly, though the effects of clinical patient characteristics (e.g. comorbidities, duration of AF and use of drugs) on the analysis of the RNA network were considered during patient selection, it is not possible to disregard these effects. Secondly, the circRNA data for NPAF and normal tissues were obtained from RNA sequencing, although further validation is required, including through the use of knockdown and overexpression experiments on target circRNAs and miRNAs.

\section{Acknowledgements}

Not applicable.

\section{Funding}

The present study was funded by the General Program of the National Natural Science Foundation of China (grant nos. 81500252 and 81770267, to DL), and the Outstanding Young Talent Training Program of Shanghai Municipal Commission of Health and Family Planning (grant no. 2017YQ045, to DL).

\section{Availability of data and materials}

The datasets used and/or analyzed during the present study are available from the corresponding author on reasonable request.

\section{Authors' contributions}

CG and YLu conceived and designed the experiments. YZ, XK and JL performed the experiments. XM, YLi, DL and LW analyzed the data. YZ, CG and YLu wrote the paper. All authors read and approved the final manuscript.

\section{Ethics approval and consent to participate}

Written informed consent from patients was obtained prior to collection of the left atrial appendages, which were abandoned due to the surgical technique. The study was approved by the ethics committees (no. 040-2017) in accordance with the relevant guidelines and regulations (clinical research registration no. ChiCTR-RRC-17014230). The patients provided written informed consent.

\section{Patient consent for publication}

The patients provided written informed consent for publication.

\section{Competing interests}

The authors declare that they have no competing interests.

\section{References}

1. Nattel S: New ideas about atrial fibrillation 50 years on. Nature 415: 219-226, 2002.

2. Gudbjartsson DF, Arnar DO, Helgadottir A, Gretarsdottir S, Holm H, Sigurdsson A, Jonasdottir A, Baker A, Thorleifsson G, Kristjansson K, et al: Variants conferring risk of atrial fibrillation on chromosome 4q25. Nature 448: 353-357, 2007.

3. Du X, Dong J and Ma C: Is atrial fibrillation a preventable disease? J Am Coll Cardiol 69: 1968-1982, 2017.

4. Chugh SS, Havmoeller R, Narayanan K, Singh D, Rienstra M, Benjamin EJ, Gillum RF, Kim YH, McAnulty JH Jr, Zheng ZJ, et al: Worldwide epidemiology of atrial fibrillation: A Global Burden of Disease 2010 Study. Circulation 129: 837-847, 2014.

5. Hijazi Z, Oldgren J, Lindbäck J, Alexander JH, Connolly SJ, Eikelboom JW, Ezekowitz MD, Held C, Hylek EM, Lopes RD, et al: A biomarker-based risk score to predict death in patients with atrial fibrillation: The $\mathrm{ABC}$ (age, biomarkers, clinical history) death risk score. Eur Heart J 39: 477-485, 2018.

6. Schnabel RB, Yin X, Gona P, Larson MG, Beiser AS, McManus DD, Newton-Cheh C, Lubitz SA, Magnani JW, Ellinor PT, et al: 50 year trends in atrial fibrillation prevalence, incidence, risk factors, and mortality in the Framingham Heart Study: A cohort study. Lancet 386: 154-162, 2015.

7. Kirchhof P: The future of atrial fibrillation management: Integrated care and stratified therapy. Lancet 390: 1873-1887, 2017.

8. Kotecha D, Calvert M, Deeks JJ, Griffith M, Kirchhof P, Lip GY, Mehta S, Slinn G, Stanbury M, Steeds RP and Townend JN: A review of rate control in atrial fibrillation, and the rationale and protocol for the RATE-AF trial. BMJ Open 7: e015099, 2017.

9. Kirchhof P, Benussi S, Kotecha D, Ahlsson A, Atar D, Casadei B, Castella M, Diener HC, Heidbuchel H, Hendriks J, et al: 2016 ESC guidelines for the management of atrial fibrillation developed in collaboration with EACTS. Europace 18: 1609-1678, 2016.

10. Chiang CE, Wang KL and Lip GY: Stroke prevention in atrial fibrillation: An Asian perspective. Thromb Haemost 111: 789-797, 2014.

11. Fauchier L, Philippart R, Clementy N, Bourguignon T, Angoulvant D, Ivanes F, Babuty D and Bernard A: How to define valvular atrial fibrillation? Arch Cardiovasc Dis 108: 530-539, 2015.

12. Boriani G, Cimaglia P, Fantecchi E, Mantovani V, Ziacchi M, Valzania C, Martignani C, Biffi $M$ and Diemberger I: Non-valvular atrial fibrillation: Potential clinical implications of the heterogeneous definitions used in trials on new oral anticoagulants. J Cardiovasc Med (Hagerstown) 16: 491-496, 2015.

13. Nigro JM, Cho KR, Fearon ER, Kern SE, Ruppert JM, Oliner JD, Kinzler KW and Vogelstein B: Scrambled exons. Cell 64: 607-613, 1991.

14. Hansen TB, Jensen TI, Clausen BH, Bramsen JB, Finsen B, Damgaard CK and Kjems J: Natural RNA circles function as efficient microRNA sponges. Nature 495: 384-388, 2013.

15. Memczak S, Jens M, Elefsinioti A, Torti F, Krueger J, Rybak A, Maier L, Mackowiak SD, Gregersen LH, Munschauer M, et al: Circular RNAs are a large class of animal RNAs with regulatory potency. Nature 495: 333-338, 2013.

16. Cortés-López M and Miura P: Emerging functions of circular RNAs. Yale J Biol Med 89: 527-537, 2016.

17. He L, Zhang A, Xiong L, Li Y, Huang R, Liao L, Zhu Z and Wang AY: Deep circular RNA sequencing provides insights into the mechanism underlying grass carp reovirus infection. Int $\mathrm{J}$ Mol Sci 18: pii: E1977, 2017.

18. Zhang S, Zhu DN, Li H, Li H, Feng C and Zhang W: Characterization of circRNA-associated-ceRNA networks in a senescence-accelerated mouse prone 8 brain. Mol Ther 25: 2053-2061, 2017. 
19. Nan A, Chen LJ, Zhang N, Liu Z, Yang T, Wang Z, Yang C and Jiang Y: A novel regulatory network among LncRpa, CircRar1, MiR-671 and apoptotic genes promotes lead-induced neuronal cell apoptosis. Arch Toxicol 91: 1671-1684, 2017.

20. Audic S and Claverie JM: Self-identification of protein-coding regions in microbial genomes. Proc Natl Acad Sci USA 95: 10026-10031, 1998.

21. Livak KJ and Schmittgen TD: Analysis of relative gene expression data using real-time quantitative PCR and the 2(-Delta Delta C(T)) method. Methods 25: 402-408, 2001.

22. Su H, Lin F, Deng X, Shen L, Fang Y, Fei Z, Zhao L, Zhang X Pan H, Xie D, et al: Profiling and bioinformatics analyses reveal differential circular RNA expression in radioresistant esophageal cancer cells. J Transl Med 14: 225, 2016.

23. Qu S, Yang X, Li X, Wang J, Gao Y, Shang R, Sun W, Dou K and Li H: Circular RNA: A new star of noncoding RNAs. Cancer Lett 365: 141-148, 2015.

24. Jeck WR and Sharpless NE: Detecting and characterizing circular RNAs. Nat Biotechnol 32: 453-461, 2014.

25. Chen LL and Yang L: Regulation of circRNA biogenesis. RNA Biol 12: 381-388, 2015.

26. Jeck WR, Sorrentino JA, Wang K, Slevin MK, Burd CE, Liu J, Marzluff WF and Sharpless NE: Circular RNAs are abundant, conserved, and associated with ALU repeats. RNA 19: 141-157, 2013.

27. Starke S, Jost I, Rossbach O, Schneider T, Schreiner S, Hung LH and Bindereif A: Exon circularization requires canonical splice signals. Cell Rep 10: 103-111, 2015.

28. Guo JN, Li J, Zhu CL, Feng WT, Shao JX, Wan L, Huang MD and He JD: Comprehensive profile of differentially expressed circular RNAs reveals that hsa_circ_0000069 is upregulated and promotes cell proliferation, migration, and invasion in colorectal cancer. Onco Targets Ther 9: 7451-7458, 2016.

29. Galasso M, Costantino G, Pasquali L, Minotti L, Baldassari F, Corrà $\mathrm{F}$, Agnoletto $\mathrm{C}$ and Volinia $\mathrm{S}$ : Profiling of the predicted circular RNAs in ductal in situ and invasive breast cancer: A pilot study. Int J Genomics 2016: 4503840, 2016.

30. Chen J, Li Y, Zheng Q, Bao C, He J, Chen B, Lyu D, Zheng B, $\mathrm{Xu} Y$, Long Z, et al: Circular RNA profile identifies circPVT1 as a proliferative factor and prognostic marker in gastric cancer. Cancer Lett 388: 208-219, 2017.

31. Tang CM, Zhang M, Huang L, Hu ZQ, Zhu JN, Xiao Z, Zhang Z, Lin QX, Zheng XL, Yang M, et al: CircRNA_000203 enhances the expression of fibrosis-associated genes by derepressing targets of miR-26b-5p, Colla2 and CTGF, in cardiac fibroblasts. Sci Rep 7: 40342, 2017.

32. Woods CE and Olgin J: Atrial fibrillation therapy now and in the future: Drugs, biologicals, and ablation. Circ Res 114: 1532-1546, 2014.

33. Brundel BJ, Van Gelder IC, Henning RH, Tuinenburg AE, Wietses M, Grandjean JG, Wilde AA, Van Gilst WH and Crijns HJ: Alterations in potassium channel gene expression in atria of patients with persistent and paroxysmal atrial fibrillation: Differential regulation of protein and mRNA levels for $\mathrm{K}+$ channels. J Am Coll Cardiol 37: 926-932, 2001.
34. Santulli G, D'ascia SL and D'ascia C: Development of atrial fibrillation in recipients of cardiac resynchronization therapy: The role of atrial reverse remodelling. Can J Cardiol 28: 245. e17-e18, 2012.

35. Xie W, Santulli G, Guo X, Gao M, Chen BX and Marks AR Imaging atrial arrhythmic intracellular calcium in intact heart. J Mol Cell Cardiol 64: 120-123, 2013.

36. Andrade J, Khairy P, Dobrev D and Nattel S: The clinical profile and pathophysiology of atrial fibrillation: Relationships among clinical features, epidemiology, and mechanisms. Circ Res 114: 1453-1468, 2014

37. Dobrev D and Nattel S: Calcium handling abnormalities in atrial fibrillation as a target for innovative therapeutics. J Cardiovase Pharmacol 52: 293-299, 2008.

38. Luo X, Pan Z, Shan H, Xiao J, Sun X, Wang N, Lin H, Xiao L, Maguy A, Qi XY, et al: MicroRNA-26 governs profibrillatory inward-rectifier potassium current changes in atrial fibrillation. J Clin Invest 123: 1939-1951, 2013.

39. Cañón S,Caballero R,Herraiz-MartinezA,Pérez-HernándezM, López B, Atienza F, Jalife J, Hove-Madsen L, Delpón E and Bernad A: miR-208b upregulation interferes with calcium handling in HL-1 atrial myocytes: Implications in human chronic atrial fibrillation. J Mol Cell Cardiol 99: 162-173, 2016.

40. Lai LP, Su MJ, Lin JL, Lin FY, Tsai CH, Chen YS, Huang SK, Tseng YZ and Lien WP: Down-regulation of L-type calcium channel and sarcoplasmic reticular $\mathrm{Ca}(2+)$-ATPase mRNA in human atrial fibrillation without significant change in the mRNA of ryanodine receptor, calsequestrin and phospholamban: An insight into the mechanism of atrial electrical remodeling. J Am Coll Cardiol 33: 1231-1237, 1999.

41. Tao H, Zhang M, Yang JJ and Shi KH: MicroRNA-21 via dysregulation of WW domain-containing protein 1 regulate atrial fibrosis in atrial fibrillation. Heart Lung Circ 27: 104-113, 2018.

42. Wang H, Bei Y, Shen S, Huang P, Shi J, Zhang J, Sun Q, Chen Y, Yang Y, Xu T, et al: miR-21-3p controls sepsis-associated cardiac dysfunction via regulating SORBS2. J Mol Cell Cardiol 94: 43-53, 2016

43. Yan MW, Chen C, Gong W, Yin Z, Zhou L, Chaugai S and Wang DW: miR-21-3p regulates cardiac hypertrophic response by targeting histone deacetylase-8. Cardiovasc Res 105: 340-352, 2015.

44. Girmatsion Z, Biliczki P, Bonauer A, Wimmer-Greinecker G, Scherer M, Moritz A, Bukowska A, Goette A, Nattel S, Hohnloser SH and Ehrlich JR: Changes in microRNA-1 expression and I-K1 up-regulation in human atrial fibrillation. Heart Rhythm 6: 1802-1809, 2009.

45. Ehrlich JR: Inward rectifier potassium currents as a target for atrial fibrillation therapy. J Cardiovasc Pharmacol 52: 129-135, 2008.

46. Doss JF, Corcoran DL, Jima DD, Telen MJ, Dave SS and Chi JT: A comprehensive joint analysis of the long and short RNA transcriptomes of human erythrocytes. BMC Genomics 16: 952 , 2015. 